\title{
Estimación de carbono almacenado en bosques de oyamel y ciprés en Texcoco, Estado de México
}

\author{
Estimation of carbon stored in fir and cypress forests in Texcoco, State of Mexico
}

\author{
Yunuen Bolaños González ${ }^{1 *}$, Martín A. Bolaños González ${ }^{3}$, \\ Fernando Paz Pellat ${ }^{2}$ y José Ignacio Ponce Pulido ${ }^{3}$
}

\footnotetext{
${ }^{1}$ Universidad Autónoma Chapingo. km 38.5 Carretera México-Texcoco. 56230 Texcoco, Estado de México, México.

* Autor responsable (yunuenbg90@gmail.com)

${ }^{2}$ Colegio de Postgraduados, Campus Montecillo. km 36.5 Carretera México-Texcoco. 56230 Montecillo, Estado de México, México.

${ }^{3}$ Programa Mexicano del Carbono. Chiconautla No.8, Col. Lomas de Cristo. 56225 Texcoco, Estado de México, México.
}

\section{RESUMEN}

Las emisiones de carbono (C) a la atmósfera, así como sus reservorios, en el sector de agricultura, silvicultura y otros usos del suelo son significativas en el balance global del ciclo del carbono; sin embargo, a pesar de esta importancia, es el sector con la mayor incertidumbre en el balance global debido principalmente a la falta de conocimiento preciso de los factores de emisión, por lo que se requiere mayor investigación en el tema. Debido a lo anterior, este trabajo tuvo como objetivo estimar el $\mathrm{C}$ almacenado en los cinco depósitos considerados en ecosistemas forestales: biomasa viva sobre el suelo, biomasa viva debajo del suelo, madera muerta sobre el suelo, mantillo y carbono orgánico del suelo (COS) en dos sitios de muestreo ubicados en las faldas del Monte Tláloc, Texcoco, Estado de México: el primero en un bosque de oyamel (Abies religiosa) y el segundo en un bosque de ciprés (Cupressus lusitanica). El promedio de la densidad de carbono en el bosque de oyamel en los cinco depósitos estimados fue de $376 \mathrm{Mg} \mathrm{ha}^{-1}$, siendo el depósito más importante el de biomasa aérea (59\%), seguido del COS (22\%); en tanto que en el bosque de ciprés fue de $205 \mathrm{Mg} \mathrm{ha}^{-1}$, siendo el depósito con mayor densidad el COS (50\%), seguido de biomasa aérea (36\%). Los valores de densidad de carbono para biomasa aérea en el caso de bosque de oyamel fueron superiores a los reportados en otros trabajos; lo cual se explica por la existencia de árboles de grandes dimensiones de esta especie en el sitio de muestreo, lo cual nos da una idea precisa del potencial como almacén de carbono que este tipo de vegetación tiene cuando llegan a estados maduros.

Cita recomendada:

Bolaños González, Y., M. A. Bolaños González, F. Paz Pellat y J. I. Ponce Pulido. 2017. Estimación de carbono almacenado en bosques de oyamel y ciprés en Texcoco, Estado de México. Terra Latinoamericana 35: 73-86.
Palabras clave: depósitos de carbono, biomasa, Abies religiosa, Cupressus lusitanica.

\section{SUMMARY}

Carbon emissions (C) to the atmosphere, as well as their reservoirs, in the sector of agriculture, forestry and other land uses are significant in the global carbon budget. However, despite their importance, it is the sector with the greatest uncertainty in the global budget due mainly to the lack of precise knowledge of emission factors, so that more research on the subject is required. Due to the above, this paper aims to measure the carbon (C) storage capacity in the five pools considered in forest ecosystems: aboveground biomass, belowground biomass, dead wood aboveground, litter and soil organic carbon (COS) from two sampling sites located in the foothills of Mount Tlaloc, Texcoco, State of Mexico: the first in a fir forest (Abies religiosa) and the second in a white cedar forest (Cupressus lusitanica). In the fir forest the average carbon density contained in the five pools estimated was $376 \mathrm{Mg} \mathrm{ha}^{-1}$; the most important was aboveground biomass (59\%), followed by SOC $(22 \%)$. In contrast, in the white cedar forest it was $205 \mathrm{Mg} \mathrm{ha}^{-1}$, with the highest density storage in SOC $(50 \%)$, followed by aboveground biomass $(36 \%)$. The values of carbon density for aboveground biomass in the fir forest were higher than those reported for similar vegetation types in other papers and is explained by the existence of large trees of this species in the sampling site. The results give us a precise idea of the potential this type of vegetation has as a carbon pool when it reaches mature states. 
Index words: carbon pools, biomass, Abies religiosa, Cupressus lusitanica.

\section{INTRODUCCIÓN}

El cambio climático global asociado al aumento potencial de la temperatura superficial del planeta es un problema que se acentúa por el rápido incremento actual en las emisiones de Gases de Efecto Invernadero (GEI) (Bolin et al., 1989) y por las dificultades de reducir en forma sustantiva el incremento de los mismos en el futuro próximo (Houghton et al., 1995); así, por ejemplo, se calcula un ingreso antropogénico a la atmósfera de aproximadamente $8.9 \mathrm{Pg} \mathrm{C}_{\mathrm{año}}{ }^{-1} \mathrm{a}$ nivel mundial (Ciais et al., 2013). Diferentes autores: Schneider (1989), Houghton y Woodwell (1989), Lashof y Ahuja (1990), Dixon et al. (1994), Masera (1995), Schimel (1995), (Le Quéré et al., 2009), Houghton et al. (2012), afirman que el dióxido de carbono $\left(\mathrm{CO}_{2}\right)$ es uno de los gases de efecto invernadero (GEI) más importantes y que su emisión a la atmósfera por el cambio de uso del suelo ocupa el segundo lugar a nivel mundial que, de acuerdo a las últimas estimaciones disponibles, se calcula 1.1-1.14 Pg C año-1 (Ciais et al., 2013, Houghton et al., 2012), con una fuerte contribución de las zonas tropicales.

En México, de acuerdo al Inventario Nacional de emisiones de GEI, la categoría Uso del Suelo, Cambio de Uso del Sueloy Silvicultura (USCUSS) fue en 2006 el tercero en liberación de GEI a la atmósfera con un $9.9 \%$ del total estimado en el país (SEMARNAT e INECC, 2009); aunque en 2010 se estimó en $6.3 \%$, siendo el cuarto en importancia (SEMARNAT e INECC, 2012), lo que representó una disminución en términos absolutos de $23310 \mathrm{Gg}$ de $\mathrm{CO}_{2}$ eq, debidos principalmente a cambios en la metodología e interpretación, dado que se utilizó la misma información base (SEMARNAT e INECC, 2012). Debido a lo anterior, los ecosistemas forestales tienen un papel prioritario en el ciclo global del carbono y en el cambio climático global, por lo que resulta de gran importancia su conservación; ya que a pesar de ello, no existen mediciones forestales colectadas en forma regular y sistemática utilizando metodologías consistentes a lo largo del tiempo y manteniendo series históricas, debido a lo cual existe un alto grado de incertidumbre en estas estimaciones (De Jong et al., 2009).
De acuerdo al Grupo Intergubernamental de Expertos sobre el Cambio Climático (IPCC, 2003 y 2006), en los ecosistemas forestales el $\mathrm{C}$ se distribuye en cinco depósitos: biomasa viva por encima del suelo (biomasa aérea), biomasa viva por debajo del suelo (biomasa subterránea), madera muerta sobre el suelo, mantillo y carbono orgánico del suelo(COS). La biomasa aérea y subterránea se definen como la cantidad total de material orgánico vivo existente, por arriba y por abajo del suelo, respectivamente, expresada como peso anhidro por unidad de área (FAO, 2004). La capacidad de los ecosistemas forestales para almacenar carbono en forma de biomasa varía en función de la composición florística, la edad y la densidad de población de cada estrato por comunidad vegetal (Schulze et al., 2000). Así, Prentice et al. (2001), estimaron que la mitad de la producción primaria bruta (PPB) se incorpora en los tejidos vegetales como hojas, raíces y tejidos leñosos (producción primaria neta o PPN), calculando globalmente con datos de los ochentas, que la PPB era de $120 \mathrm{Pg}_{\text {año }}{ }^{-1}$ de C. Posteriormente, Beer et al. (2010) estimaron la PPB en $123 \pm 8 \mathrm{Pg}$ año ${ }^{-1}$.

El COS es el producto de la descomposición de plantas, el metabolismo de organismos vivos y el crecimiento bacteriano (Gardi et al., 2014). Se estima que el depósito de COS a $1 \mathrm{~m}$ de profundidad es de 1500-2400 Pg (Batjes, 1996; Lal, 2003; Ciais et al., 2013), tres a cuatro veces mayor que el depósito de $C$ en la vegetación (550 Pg C) y dos veces mayor que el atmosférico (760 Pg) (Eswaran et al., 1993; Lal, 2003).

El mantillo incluye desde materia orgánica fresca hasta materiales orgánicos altamente descompuestos y parcialmente estabilizados y es la fuente principal de $\mathrm{C}$ y de nutrientes disponibles para las plantas (Swift et al., 1979; Caldato et al., 2010), teniendo en gran medida responsabilidad en el funcionamiento de los ecosistemas forestales. La calidad del mantillo (relación carbono: nitrógeno) está determinada en gran medida por las especies de árboles presentes, que a su vez dependen de la elevación, el clima y la historia de uso (Berg, 2000).

De acuerdo al IPCC (2006), la madera muerta comprende toda la biomasa boscosa no viva no contenida en el mantillo, ya sea en pie, superficial o en el suelo. Es un componente natural importante en los bosques, que además de ser un elemento clave de la biodiversidad forestal, también influencia 
el microclima de éstos, actúa como un almacén de agua en períodos secos (Harmon et al., 1986) y como un almacén de C y nutrientes a largo plazo (Harmon et al., 1986; Schaetzl et al., 1989; Keenan et al., 1993).

El Monte Tláloc forma parte de la Sierra Nevada, una de las regiones terrestres prioritarias para la Comisión Nacional de Áreas Naturales Protegidas (CONANP), y representa un área de gran importancia desde el punto de vista biogeográfico puesto que ahí convergen las regiones neártica y neotropical, además de valores altos de riqueza y endemismos (Arriaga, et al., 2000) y como principal zona de recarga y amortiguamiento hidrológico del Valle de México (Moctezuma y Carreón, 2005). Adicionalmente, la Sierra Nevada se sitúa al oriente de uno de los mayores complejos urbanos del mundo: la zona metropolitana de la Ciudad de México, la cual ejerce una enorme presión sobre la vegetación circundante y en general sobre los recursos naturales (Sánchez y López, 2003), misma que, se prevé, se incremente con la construcción del Nuevo Aeropuerto Internacional de la Ciudad de México (OECD, 2015), en especial en el municipio de Texcoco, Estado de México. Debido a esta importancia geográfica estratégica, a la falta de información específica de factores de emisión del sector agrícola, silvícola y otros usos del suelo (AFOLU, por sus siglas en inglés) y a la capacidad de almacenamiento de carbono de los ecosistemas forestales, descrita anteriormente; en el presente estudio se seleccionaron dos sitios de muestreo ubicados en el municipio de Texcoco, utilizando metodologías de muestreo y laboratorio estandarizadas con los criterios definidos por el IPCC (IPCC, 2003 y 2006) para la estimación de los depósitos de carbono de un bosque de oyamel y un bosque de ciprés; aportando información para reducir el grado de incertidumbre en las estimaciones de emisiones de GEI que a la fecha se tienen en este sector, por lo que este trabajo contribuye con este objetivo.

\section{MATERIALES Y MÉTODOS}

\section{Sitios de Muestreo}

El área de muestreo se ubicó entre las coordenadas $19^{\circ} 26^{\prime} 42.36^{\prime \prime}$ y $19^{\circ} 28^{\prime} 20.09^{\prime \prime} \mathrm{N}$ y $98^{\circ} 44^{\prime} 59.74$ " y $98^{\circ} 46^{\prime} 42.78^{\prime \prime}$ O e integra parte de los ejidos de Santa Catarina del Monte, San Pablo Ixayoc, Santa María Nativitas y San Miguel Tlaixpan, todos en el municipio de Texcoco. Se seleccionó un paisaje de $9 \mathrm{~km}^{2}$ espacialmente representativo de la vegetación y de los tipos de suelos de los bosques templados del oriente del Estado de México, dentro de esta área se delimitaron dos zonas denominadas Bosques de Referencia (BR), con rasgos homogéneos en términos de almacenes de carbono: el primero fue un bosque monoespecífico de oyamel (Abies religiosa) y el segundo un bosque monoespecífico de ciprés (Cupressus lusitanica). Cada BR (Conglomerado) se integró por cuatro sitios de muestreo (Figura 1). Algunas de las variables de densidad de ambos BR se muestran en el Cuadro 1, en el que se agregó como referencia el número de

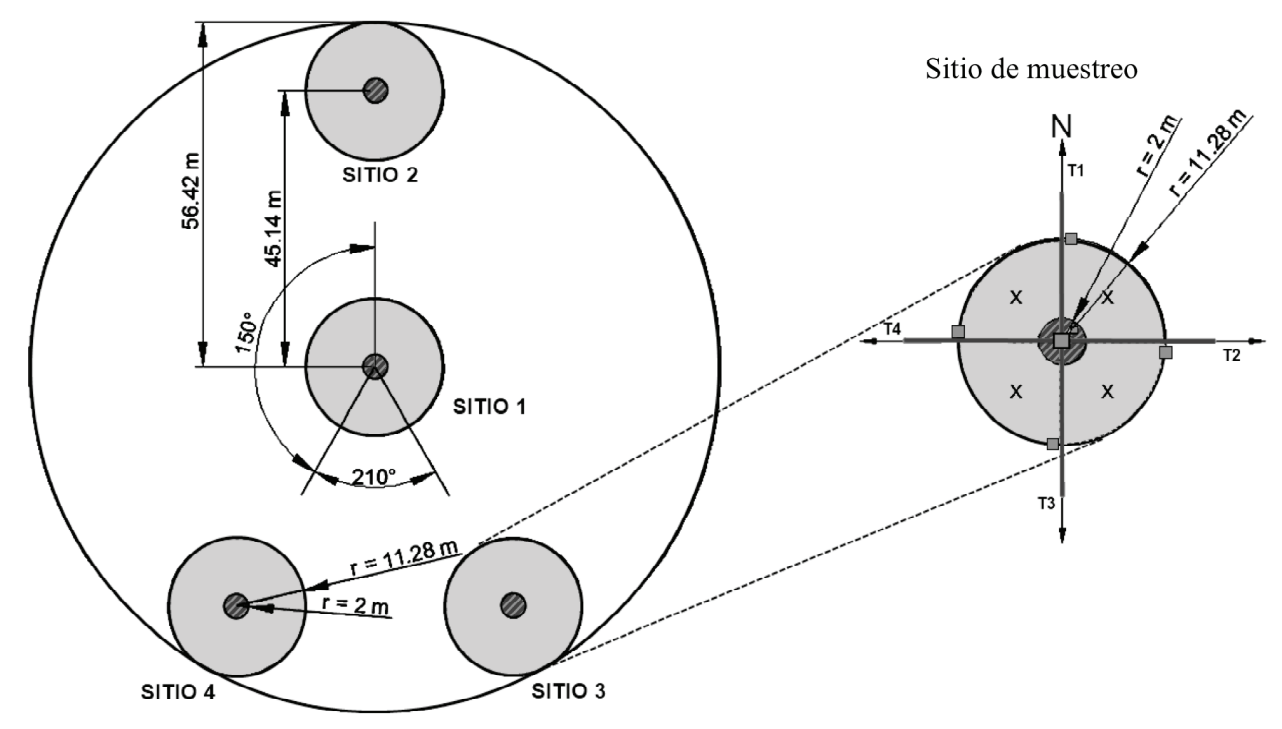

Figura 1. Diseño del conglomerado (Bosque de Referencia). Fuente: Elaboración propia, adaptado de CONAFOR 2011. 
Cuadro 1. Variables de densidad de los bosques de referencia.

\begin{tabular}{|c|c|c|c|c|c|c|}
\hline BR/Especie & Sitio & $\mathrm{NAV}^{\dagger}$ & $\begin{array}{l}\text { Frecuencia } \\
\text { relativa }\end{array}$ & Área basal & NAMP & $\begin{array}{l}\text { Número de } \\
\text { tocones }\end{array}$ \\
\hline & & & $\%$ & $\mathrm{~m}^{2} \mathrm{ha}^{-1}$ & & \\
\hline \multirow[t]{4}{*}{$\mathrm{BR} 1 /$ Abies religiosa } & 1 & 10 & 91 & 30.24 & 1 & 9 \\
\hline & 2 & 8 & 100 & 40.23 & 2 & 10 \\
\hline & 3 & 8 & 100 & 131.25 & 0 & 5 \\
\hline & 4 & 12 & 100 & 231.07 & 0 & 12 \\
\hline \multirow[t]{4}{*}{ BR2/Cupressus lusitanica } & 1 & 33 & 100 & 46.85 & 1 & 91 \\
\hline & 2 & 23 & 100 & 48.43 & 2 & 29 \\
\hline & 3 & 46 & 98 & 48.23 & 2 & 90 \\
\hline & 4 & 47 & 98 & 42.20 & 0 & 83 \\
\hline
\end{tabular}

${ }^{\dagger} \mathrm{NAV}=$ número de árboles vivos de la especie dominante con $\mathrm{DN} \geq 7.5 \mathrm{~cm} .{ }^{\star} \mathrm{NAMP}=$ número de árboles muertos en pie.

árboles muertos en pie y tocones medidos en ellos. Este último dato muestra que ambos BR han estado sujetos a intervención humana, dado que en la mayoría de sitios que conforman cada BR el número de tocones encontrados fue superior al de árboles vivos. Destaca también el hecho de que el área basal de los sitios 3 y 4 del BR1 es mayor que la de los sitios 1 y 2 del mismo BR a pesar de tener una cantidad similar de individuos.

Respecto a las clases diamétricas, el BR1 (oyamel) corresponde a una masa forestal irregular, con un rango de clases muy amplio. En el BR2 (ciprés) los individuos se concentran en las clases más pequeñas $(10-50 \mathrm{~cm})$, por lo que a pesar de tener una mayor cantidad de individuos con respecto a los sitios 3 y 4 del BR1, su área basal es sensiblemente inferior a éstos (Cuadro 2).

\section{Diseño del Muestreo de Campo}

El diseño del muestreo de campo se orientó para obtener datos cuantitativos de COS, mantillo, madera muerta, biomasa área y biomasa subterránea. Los sitios cuantitativos se distribuyeron en los BR formando un conglomerado tipo CONAFOR integrado por cuatro sitios de muestreo circulares de $400 \mathrm{~m}^{2}$ de área en forma de "Y" invertida (CONAFOR, 2011) (Figura 1).

En cada uno de los cuatro sitios cuantitativos del conglomerado se midieron todos los árboles con diámetro normal $(\mathrm{DN}) \geq 7.5 \mathrm{~cm}$, los arbustos y se tomaron cuatro muestras de biomasa de herbáceas $\left(0.25 \mathrm{~m}^{2}\right)$, mismas que se colectaron en áreas representativas de cada sitio. Se colectaron cinco muestras de mantillo

Cuadro 2. Clases diamétricas de los bosques de referencia (BR).

\begin{tabular}{|c|c|c|c|c|c|c|c|c|c|c|c|c|c|c|c|c|c|}
\hline \multirow[t]{3}{*}{ BR/Especie } & \multirow[t]{3}{*}{ Sitio } & \multicolumn{16}{|c|}{ Frecuencia absoluta por clase diamétrica } \\
\hline & & \multicolumn{16}{|c|}{ - $\ldots$} \\
\hline & & 10 & 15 & 20 & 25 & 30 & 35 & 40 & 45 & 50 & 55 & 60 & 65 & 70 & 75 & 80 & $>80$ \\
\hline \multirow[t]{4}{*}{$\mathrm{BR} 1 /$ Abies religiosa } & 1 & 0 & 1 & 1 & 0 & 1 & 2 & 2 & 1 & 1 & 0 & 1 & 0 & 0 & 0 & 0 & 0 \\
\hline & 2 & 0 & 0 & 0 & 0 & 0 & 2 & 1 & 2 & 1 & 1 & 0 & 0 & 0 & 0 & 1 & 0 \\
\hline & 3 & 1 & 1 & 1 & 0 & 0 & 2 & 0 & 1 & 0 & 0 & 0 & 0 & 0 & 0 & 0 & 2 \\
\hline & 4 & 1 & 3 & 0 & 0 & 0 & 0 & 0 & 0 & 0 & 4 & 1 & 0 & 0 & 0 & 0 & 3 \\
\hline \multirow[t]{4}{*}{ BR2/Cupressus lusitanica } & 1 & 7 & 3 & 2 & 7 & 8 & 2 & 2 & 1 & 1 & 0 & 0 & 0 & 0 & 0 & 0 & 0 \\
\hline & 2 & 0 & 2 & 2 & 6 & 0 & 4 & 5 & 2 & 1 & 0 & 0 & 0 & 0 & 0 & 0 & 0 \\
\hline & 3 & 17 & 9 & 3 & 5 & 5 & 2 & 2 & 2 & 1 & 0 & 0 & 0 & 0 & 0 & 0 & 0 \\
\hline & 4 & 16 & 8 & 5 & 11 & 4 & 3 & 1 & 0 & 0 & 0 & 0 & 0 & 0 & 0 & 0 & 0 \\
\hline
\end{tabular}


(hojarasca y capa de fermentación) y suelo, cuatro de ellas distribuidas en la periferia del círculo que delimitó el sitio, orientadas a cada uno de los puntos cardinales, y una en el centro del mismo, denominada DAP (donde se midió densidad aparente del suelo). Finalmente, se trazaron dos transectos perpendiculares entre sí para medir el material leñoso caído (combustibles), tal como se muestra en la Figura 2.

\section{Estimación de Depósitos de Carbono}

Los depósitos considerados fueron los siguientes:

Biomasa aérea. Se refiere a toda la biomasa viva por encima del suelo incluyendo el fuste, las ramas, la corteza, las semillas y las hojas; su cuantificación es de gran importancia debido a que aproximadamente el 50\% de ella es C (Brown, 1997; Chojnacky y Milton, 2008). En el caso de la vegetación arbórea se midieron los datos de diámetro normal (DN) de cada árbol registrado por sitio de muestreo. Con estos datos se realizó el cálculo de biomasa para Abies religiosa utilizando ecuaciones alométricas y factores de expansión de biomasa encontrados en la literatura revisada (Cuadro 3).
Basado en los resultados obtenidos en el cálculo de biomasa de Abies religiosa, para cuantificar el contenido de carbono se seleccionaron dos ecuaciones alométricas para esta especie, tomando como criterio de selección el que se explica en la sección denominada Estimación de contenido de biomasa de Abies religiosa. Adicionalmente se utilizó una ecuación para el cálculo de biomasa y carbono de Cupressus lusitanica y otra para Quercus laurina (se encontraron 2 árboles de esta especie en el BR2 y uno en el BR1), las cuales corresponden a las especies arbóreas encontradas en los sitios de muestreo (Cuadro 4), tomando como criterios de selección el que corresponden a la misma especie y que fueron obtenidas en sitios próximos geográficamente al área de estudio.

En lo que respecta a la vegetación arbustiva se realizó un procedimiento similar al usado para la vegetación arbórea, pero no se encontraron en la literatura ecuaciones alométricas desarrolladas para las especies encontradas en los sitios de muestreo, por lo que se utilizó la ecuación desarrollada por Búrquez et al. (2010), ya que, aunque fue desarrollada para especies de zonas desérticas (la gran mayoría de ecuaciones encontradas fueron desarrolladas para especies de éstas zonas, donde el aporte del estrato arbustivo a la biomasa

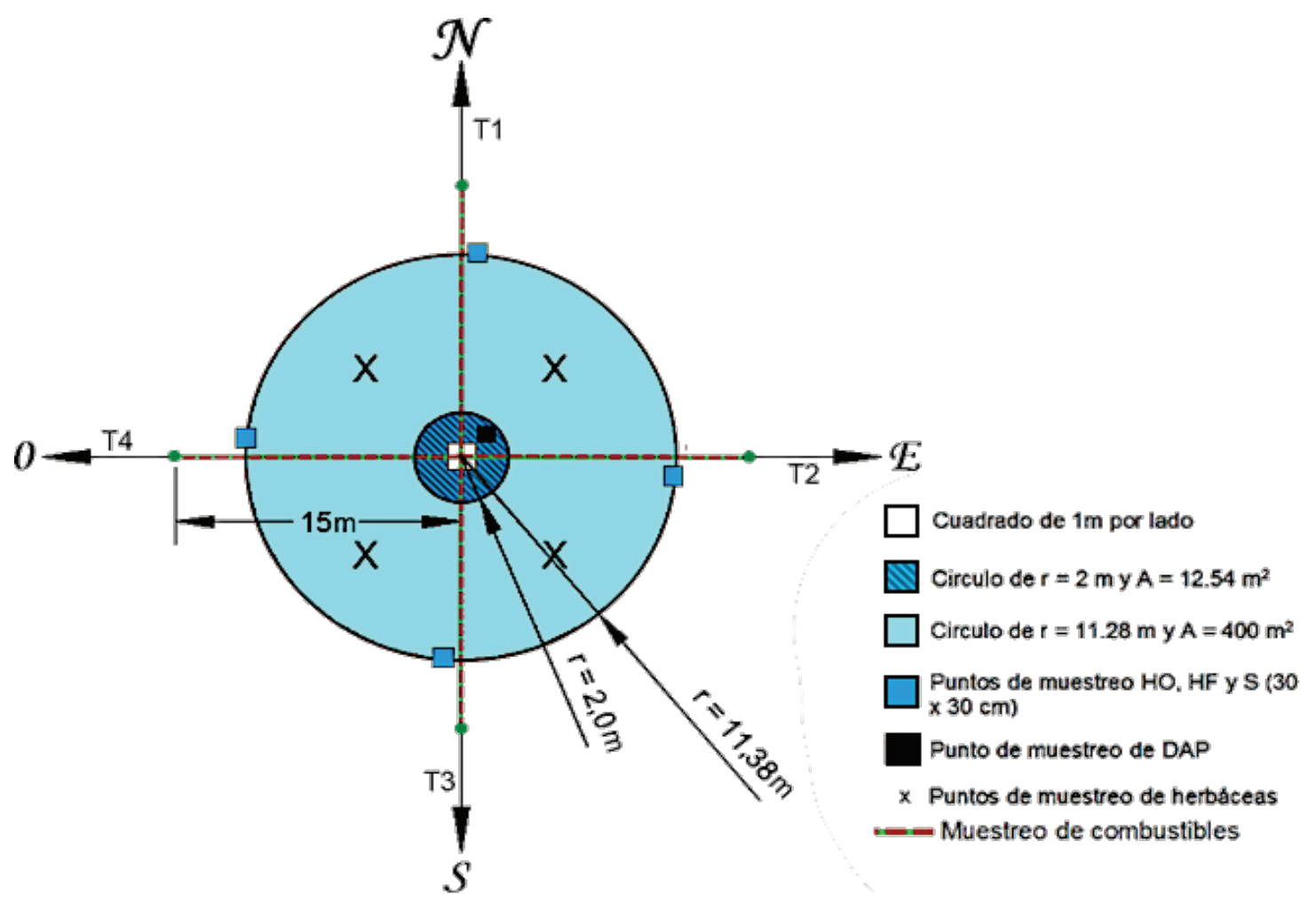

Figura 2. Diseño del sitio de muestreo cuantitativo y puntos de toma de datos. 
Cuadro 3. Métodos/modelos para estimación de biomasa de Abies religiosa.

\begin{tabular}{|c|c|c|}
\hline Autor & Modelo/Método & Observaciones \\
\hline $\begin{array}{l}\text { Avendaño } \\
\text { et al., } 2009\end{array}$ & $B=0.0713 D N^{2.5104}$ & $\begin{array}{l}\text { Ecuación utilizada para estimar la biomasa de árboles individuales en } \\
\text { Nanacamilpa, Tlaxcala. El modelo se generó con datos de árboles con } \\
\text { diámetro normal }(\mathrm{DN}) \text { de } 6.5-79 \mathrm{~cm} \text {. }\end{array}$ \\
\hline $\begin{array}{l}\text { Brown et al., } \\
1997\end{array}$ & $B=\frac{0.5+15000 D N^{2.7}}{D N^{2.7}+364946}$ & $\begin{array}{l}\text { Ecuación utilizada para estimar la biomasa de especies de coníferas de } \\
\text { bosques no perturbados en Estados Unidos, incluyendo árboles de grandes } \\
\text { dimensiones }(\mathrm{DN}>70 \mathrm{~cm}) \text {. El DN está en centímetros y la } \mathrm{B} \text { en } \mathrm{kg}^{-1} \text { abol }^{-1} \text {. }\end{array}$ \\
\hline $\begin{array}{l}\text { Flores et al., } \\
2011\end{array}$ & $\begin{array}{c}B_{f}=0.0173 D N^{2.7459} \\
+ \\
B_{m r a}=\begin{array}{c}1.5842 e^{0.044 D N} \\
+\end{array} \\
B_{f a}=0.8413 e^{0.0398 D N}\end{array}$ & $\begin{array}{l}\text { Ecuaciones para estimar biomasa de árboles individuales en bosques en } \\
\text { decaimiento en el Desierto de los Leones (D.F.) y Monte Tláloc (Edo. de } \\
\text { México), en tres secciones: biomasa de fuste }\left(\mathrm{B}_{\mathrm{f}}\right) \text {, biomasa de madera de } \\
\text { ramas }\left(\mathrm{B}_{\mathrm{mra}}\right) \text { y biomasa de acículas }\left(\mathrm{B}_{\mathrm{fa}}\right) \text {. El modelo se generó con datos de } \\
\text { árboles con DN de } 12-105 \mathrm{~cm} \text {. }\end{array}$ \\
\hline $\begin{array}{l}\text { Rodríguez y } \\
\text { Calva, } 2013\end{array}$ & $B=0.0519 D N^{2.5954}$ & $\begin{array}{l}\text { Ecuación utilizada para estimar biomasa de árboles individuales en el Parque } \\
\text { Nacional El Chico, Hidalgo; la cual se generó con datos de árboles con DN de } \\
2.86-100.59 \mathrm{~cm} \text {. }\end{array}$ \\
\hline $\begin{array}{l}\mathrm{FEB}^{\dagger} \\
\text { IPCC, } 2003\end{array}$ & $\begin{array}{l}{ }^{\infty} B=V_{f} \cdot \delta_{b} \cdot F E B \\
{ }^{\circ} V_{f}=\frac{\pi D N^{2}}{4} \cdot h \cdot C M\end{array}$ & $\begin{array}{l}\text { El volumen del fuste }\left(\mathrm{V}_{\mathrm{f}}\right) \text { se calculó con un coeficiente mórfico forestal }(\mathrm{CM}) \\
\text { de } 0.7 \text { y se utilizó una densidad básica de la madera }\left(\delta_{\mathrm{b}}\right) \text { de } 0.3874 \text { y un FEB } \\
\text { de } 1.3 \text {, valores tomados de Rojas y Villers }(2008) \text {. }\end{array}$ \\
\hline
\end{tabular}

total del sitio es muy importante) mostró consistencia en las estimaciones de biomasa de muchas especies con diferentes formas de crecimiento, tamaños, condiciones edáficas y de disponibilidad de humedad.

Así, el modelo utilizado considera el área proyectada de la copa como una elipse, de tal forma que el volumen de ésta corresponde a un cilindro elíptico, Ecuación 1.

$V=\pi \times r_{1} \times r_{2} \times h$

De los tres modelos desarrollados por Búrquez et al. (2010), en este trabajo se seleccionó el no lineal, mismo que se generó en condiciones de mayor disponibilidad de agua en el suelo y estima la cantidad de biomasa seca sobre el mismo $\left(\mathrm{Bm}, \mathrm{g} \mathrm{m}^{-2}\right)$ a partir del volumen de copa $\left(\mathrm{V}\right.$, en $\left.\mathrm{m}^{3} \mathrm{~m}^{-2}\right)$ con la Ecuación 2.

$B m=356.983 V^{1.416}$

El contenido de carbono en el estrato herbáceo se calculó a partir del peso seco de la muestra $\left(1 \mathrm{~m}^{2}\right)$ y se obtuvo la densidad de la biomasa $\left(\mathrm{Mg} \mathrm{ha}^{-1}\right)$. Posteriormente el valor de densidad de biomasa se multiplicó por el factor de contenido de carbono recomendado por el IPCC (2003) de 0.5 para obtener la densidad de carbono en este estrato.

Biomasa subterránea. Este reservorio incluye toda la biomasa de raíces vivas excluyendo raíces finas de 
Cuadro 4. Ecuaciones alométricas utilizadas para la estimación de contenido de carbono en biomasa aérea arbórea.

\begin{tabular}{lccl}
\hline Especie & Ecuación para biomasa & Ecuación para carbono & Referencia bibliográfica \\
\hline $\begin{array}{l}\text { A. religiosa } \\
\text { A. religiosa }\end{array}$ & $B=0.0713 D N^{2.5104}$ & $C=0.0332 D N^{2.5103}$ & Avendaño et al., 2009. \\
& $B=\frac{0.5+15000 D N^{2.7}}{D N^{2.7}+364946}$ & $C=0.465 * B$ & $\begin{array}{l}\text { Brown et al., 1997. Se tomó el valor de contenido } \\
\text { de carbono (CC) de } 46.5 \% \text { (Avendaño et al., } \\
2009) .\end{array}$ \\
$\begin{array}{l}\text { Cupressus } \\
\text { lusitanica }\end{array}$ & $C=0.2639 D N^{1.7698}$ & FAO, 2013. \\
$\begin{array}{l}\text { Quercus } \\
\text { laurina }\end{array}$ & $B=0.1033 D N^{2.39}$ & $C=0.5 * B$ & Acosta et al., 2002. Se tomó el valor de contenido \\
& & & de carbono (CC) de 50\% (IPCC, 2003). \\
\hline
\end{tabular}

menos de $2 \mathrm{~mm}$ de diámetro debido a que con frecuencia no se pueden distinguir de la materia orgánica del suelo o mantillo (IPCC, 2006). En el presente estudio este depósito se estimó únicamente para vegetación arbórea viva, ya que se consideró que la de la vegetación herbácea es poco significativa y la de la vegetación arbustiva requiere realizar muestreos destructivos, que implican una gran cantidad de tiempo y recursos económicos (no son aplicables las ecuaciones alométricas existentes en la literatura), además de provocar una perturbación mayor en los sitios de medición, por lo que se optó por no considerarlos en la cuantificación de éste depósito. Basado en el trabajo realizado por Cairns et al. (1997), el IPCC (2006) recomienda la utilización de la ecuación alométrica (Ecuación 3) para estimar la biomasa por debajo del suelo en bosques templados, en función de la biomasa aérea seca (BSS) calculada previamente.

$\mathrm{Y}=\mathrm{e}^{[-1.0587+0.8836 \cdot \operatorname{In}(\mathrm{BSS})+0.2840]}$

Carbono orgánico del suelo (COS). El COS se estimó en función de la densidad aparente, profundidad, fracción gruesa del suelo (partículas mayores a $2 \mathrm{~mm}$ ) y porcentaje de carbono medido con un analizador automático Shimadzu 5050A. Para obtener el valor del COS se utilizó la Ecuación 4.

$$
C O S=D A P \cdot P \bullet 10000 \bullet(1-F G 100) \cdot \operatorname{COS}_{p}(0.01)
$$

dónde:

$\mathrm{COS}=$ carbono orgánico del suelo $\left(\mathrm{Mg} \mathrm{ha}^{-1}\right)$

$\mathrm{DAP}=$ densidad aparente del suelo $\left(\mathrm{Mg} \mathrm{m}^{-3}\right)$

$\mathrm{P}=$ profundidad del suelo $(\mathrm{m})$

$\mathrm{FG}=$ fragmentos gruesos en el suelo $(>2 \mathrm{~mm})$, en $\%$

$\mathrm{COS}_{\mathrm{p}}=$ contenido de carbono orgánico, en $\%$

0.01 factor de conversión de unidades (porcentaje a fracción).

El valor del factor de escala (10 000) tiene como unidades $\mathrm{m}^{2}$ ha ${ }^{-1}$.

El valor de la DAP se obtuvo tomando una muestra de suelo en campo, misma que se llevó al laboratorio para secado en estufa y determinar su peso seco. La profundidad que se consideró en el cálculo del contenido de carbono para el presente estudio fue de $0.3 \mathrm{~m}$ debido a que, de acuerdo al IPCC (2003), es una buena práctica medir el depósito de carbono en el suelo mínimamente a esta profundidad, ya que probablemente donde se producirán las mayores variaciones es en este depósito en el tiempo. El porcentaje de FG se obtuvo secando, moliendo y tamizando el suelo de las muestras en una malla 10, con abertura de $2 \mathrm{~mm}$, calculando el peso de ambas fracciones (gruesa y fina) y su respectivo porcentaje en relación al total de la muestra. Posteriormente se obtuvo una submuestra de $5 \mathrm{~g}$, la cual se tamizó en malla 100 para estimar el $\operatorname{COS}_{\mathrm{p}}$. Mantillo. Incluyó la hojarasca, capa de fermentación y material leñoso caído fino con diámetro menor 
de $7.5 \mathrm{~cm}$. El cálculo de contenido de $\mathrm{C}$ se realizó determinando el peso seco de cada compartimento y midiendo el porcentaje de carbono de las muestras mediante el analizador automático Shimadzu 5050A.

Madera muerta. Dentro de este depósito se consideró árboles muertos en pie, tocones, trozas o material leñoso caído con un diámetro $\geq 7.5 \mathrm{~cm}$. Para los árboles se estimó el contenido de carbono utilizando las mismas ecuaciones alométricas que para biomasa aérea, para los tocones se estimó su volumen con el díametro y altura medido (asumiendo que tienen forma cilíndrica) y la densidad de madera de acuerdo a su grado de putrefacción y para el caso del material leñoso caído (MLC) se utilizó el método de líneas de intersección recomendado por el IPCC (Van Wagner, 1968; IPCC, 2006), Ecuación 5, y la densidad de madera de acuerdo a su grado de putrefacción.

$V=\frac{\pi^{2} *\left(D_{1}^{2}+D_{2}^{2}+\ldots+D_{n}^{2}\right)}{8 L}$

dónde:

$\mathrm{V}=$ volumen de $\operatorname{MLC}\left(\mathrm{m}^{3} \mathrm{ha}^{-1}\right)$

$\mathrm{D}=$ diámetro de cada una de las $\mathrm{n}$ trozas $(\mathrm{cm})$

$\mathrm{L}=$ largo de la línea (suma de todos los transectos) en sitio de muestreo (m)

Posteriormente se estimó la biomasa, para ello se utilizó la Ecuación 6.

$B_{M L C}=V^{*} D M$

dónde:

$\mathrm{B}_{\mathrm{MLC}}=$ biomasa del MLC $\left(\mathrm{Mg} \mathrm{ha}^{-1}\right)$

$\mathrm{V}=$ volumen de $\operatorname{MLC}\left(\mathrm{m}^{3} \mathrm{ha}^{-1}\right)$

$\mathrm{DM}=$ densidad de la madera $\left(\mathrm{Mg} \mathrm{m}^{-3}\right)$

\section{RESULTADOS Y DISCUSIÓN}

\section{Estimación de Contenido de Biomasa de Abies religiosa}

En el BR1 se encontraron árboles de Abies religiosa con Diámetro Normal (DN) $>70 \mathrm{~cm}$, de grandes dimensiones de acuerdo a la definición de Brown et al. (1997), mismos que estaban fuera del rango de estimación de todas las ecuaciones alométricas encontradas inicialmente para esta especie en la literatura revisada (Avendaño et al., 2009; Rodríguez y Calva, 2013; Flores et al., 2011), por lo que las estimaciones de biomasa y contenido de carbono con las ecuaciones mencionadas resultaron ser mucho mayores a todo lo reportado en la literatura para esta especie. Por lo anterior, se utilizó el método de Factores de Expansión de Biomasa (FEB), que es uno de los dos que recomienda el IPCC (2003), y la ecuación alométrica propuesta por Brown et al. (1997) utilizada en la cuantificación de contenido de carbono en bosques inalterados del este de Estados Unidos, en donde se encontraron árboles de grandes dimensiones, similares a los encontrados en el BR1. En los sitios

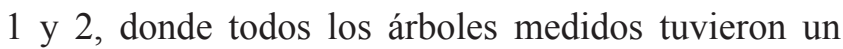
$\mathrm{DN}<70 \mathrm{~cm}$, la cantidad de biomasa estimada con todos los métodos utilizados es relativamente similar, dado que el coeficiente de variación (CV) para el sitio 1 es de $32.6 \%$ y $31.5 \%$ para el sitio 2 (Cuadro 5), siendo la ecuación que calcula el valor menor la propuesta por Flores et al. (2011) y el mayor valor al utilizar el método de factores de expansión de biomasa (IPCC, 2003). Las otras tres ecuaciones alométricas propuestas (Brown et al., 1997; Avendaño et al., 2009; Rodríguez y Calva, 2013) estiman valores muy similares de biomasa, por lo que, tomando en cuenta únicamente éstas ecuaciones, el CV se reduce a $4.32 \%$ para el sitio 1 y $1.91 \%$ para el sitio 2 (Cuadro 5).

En cambio, en los sitios 3 y 4 del BR1, donde se midieron los árboles con $\mathrm{DN}>80 \mathrm{~cm}$, existió una diferencia notable entre las estimaciones con los métodos utilizados. Así, con excepción de la ecuación alométrica propuesta por Brown et al. (1997), el resto calculó cantidades superiores a lo reportado en la literatura revisada para este tipo de vegetación y especie (De Jong et al., 1999; Ordóñez et al., 2008; Avendaño et al., 2009; Rodríguez y Calva, 2013; Flores et al., 2011). Lo anterior se debe a que los $\mathrm{DN}$ de los árboles de grandes dimensiones están fuera de los rangos utilizados en la construcción de estas ecuaciones alométricas, por lo que no consideran esta condición. Así, por ejemplo, Avendaño et al. (2009) utilizaron rangos de DN entre 5.7 y $79 \mathrm{~cm}$; pero en el BR1 se midieron tres árboles en el Sitio 4 con $\mathrm{DN}>79 \mathrm{~cm}(84,159$ y $262 \mathrm{~cm})$ y en el Sitio 3 dos árboles $(132$ y $210 \mathrm{~cm})$, por lo que el modelo utilizado no es representativo de estas clases diamétricas $\mathrm{y}$, aunque son solo cinco especímenes de un total de 38 medidos, dadas sus dimensiones, es suficiente para sobreestimar el contenido de carbono en estos sitios.

Adicionalmente, en la Figura 3 se muestra una comparación de los valores de biomasa por árbol 
Cuadro 5. Biomasa aérea de Abies religiosa calculada con diferentes métodos/ecuaciones alométricas.

\begin{tabular}{|c|c|c|c|c|c|}
\hline Modelo/Método & Sitio & Biomasa & Promedio & D. E. ${ }^{\dagger}$ & $\mathrm{CV}$ \\
\hline & & $-\cdots$ & - Mg ha-1 & --- & $\%$ \\
\hline \multirow[t]{4}{*}{ Avendaño et al., 2009} & 1 & 189 & 1289 & 1345 & 104 \\
\hline & 2 & 285 & & & \\
\hline & 3 & 1640 & & & \\
\hline & 4 & 3046 & & & \\
\hline \multirow[t]{4}{*}{ Brown et al., 1997} & 1 & 204 & 516 & 342 & 66 \\
\hline & 2 & 296 & & & \\
\hline & 3 & 601 & & & \\
\hline & 4 & 961 & & & \\
\hline \multirow[t]{4}{*}{ Rodríguez y Calva, 2013} & 1 & 190 & 1455 & 1552 & 107 \\
\hline & 2 & 292 & & & \\
\hline & 3 & 1854 & & & \\
\hline & 4 & 3484 & & & \\
\hline \multirow[t]{4}{*}{ Flores et al., 2011} & 1 & 116 & 2389 & 3429 & 144 \\
\hline & 2 & 184 & & & \\
\hline & 3 & 1867 & & & \\
\hline & 4 & 7387 & & & \\
\hline \multirow[t]{4}{*}{ Factores de expansión de biomasa (IPCC, 2003) } & 1 & 298 & 1471 & 1392 & 95 \\
\hline & 2 & 449 & & & \\
\hline & 3 & 1863 & & & \\
\hline & 4 & 3271 & & & \\
\hline
\end{tabular}

$\bar{\dagger}=$ desviación estándar; $*=$ coeficiente de variación.

estimada con las ecuaciones alométricas propuestas por Avendaño et al. (2009) y Brown et al. (1997), donde se observó que para árboles de Abies religiosa con $\mathrm{DN}<80$ $\mathrm{cm}$ de los cuatro sitios que integran el conglomerado (BR) los valores estimados son muy similares con ambas ecuaciones. En contraste, para árboles de esta misma especie, pero con $\mathrm{DN}>80 \mathrm{~cm}$, se observó una diferencia amplia en las estimaciones, misma que es mayor a medida que crece el DN (Figura 4), lo cual se debe principalmente a que la ecuación propuesta por Avendaño et al. (2009) es de tipo exponencial, por lo que los valores estimados crecen rápidamente conforme se incrementa el valor de la variable independiente (DN).

Como resultado del análisis anterior, para estimar el contenido de $\mathrm{C}$ en el BR1 se utilizó la ecuación alométrica propuesta por Avendaño et al. (2009) para árboles de $A$. religiosa con $\mathrm{DN} \leq 80 \mathrm{~cm}$, considerada más precisa debido a que es específica para esta especie y se generó a partir de un sitio geográficamente próximo y con condiciones similares al del presente estudio; y la propuesta por Brown et al. (1997) para los especímenes con DN superior a este valor: dos en el caso de sitio 3 y tres en el caso del sitio 4, como se mencionó anteriormente.

\section{Cálculo de Contenido de Carbono}

La densidad de $\mathrm{C}$ total fue mayor en el BR1 con respecto al BR2, siendo superior en todos los depósitos considerados con excepción del COS. Así, el promedio de la densidad de carbono total para los sitios del BR 1 fue de $376 \mathrm{Mg} \mathrm{ha}^{-1}$, siendo el depósito más importante el de biomasa aérea (59\%), seguido del COS (22\%); en tanto que en el BR 2 fue de $205 \mathrm{Mg} \mathrm{ha}^{-1}$, siendo el depósito con mayor densidad el COS (50\%), seguido de biomasa aérea (36\%). En el Cuadro 6 se muestran las densidades de carbono para los cinco depósitos considerados en el presente estudio, así como los promedios para cada uno de los BR medidos.

Para el caso de densidad de carbono en biomasa aérea, el contenido mayor en el BR1 con respecto 


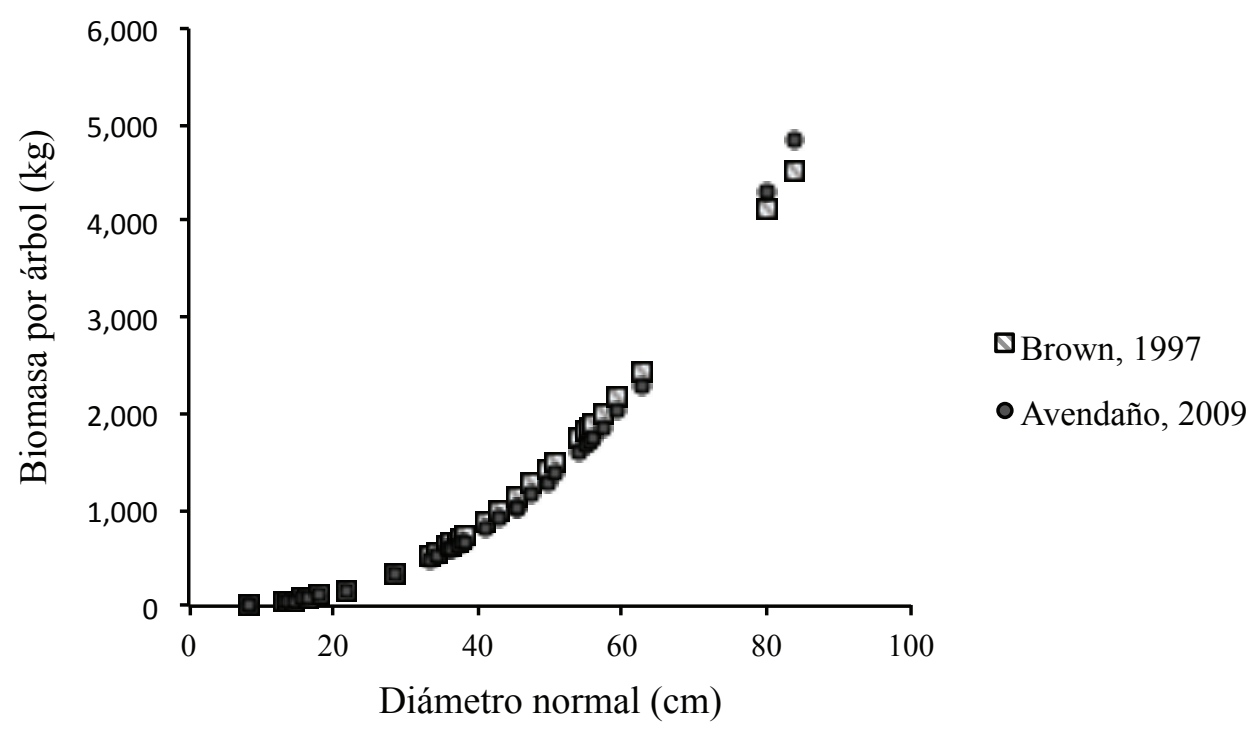

Figura 3. Biomasa aérea arbórea de Abies religiosa, utilizando las ecuaciones alométricas propuestas por Avendaño et al. (2009) y Brown et al. (1997), especímenes con $\mathrm{DN}<85 \mathrm{~cm}$.

al BR2 se debe principalmente a que las ecuaciones alométricas utilizadas están en función de la especie considerando como variable independiente el DN de los individuos, por lo que al ser mayores los DN de los especímenes de Abies religiosa medidos en el BR1, a pesar de ser menos individuos, con respecto a los especímenes de Cupressus lusitanica medidos en el $\mathrm{BR} 2$, la biomasa aérea es mayor en el primero.

En el BR1, el valor calculado de $\mathrm{C}$ en biomasa aérea fue superior al reportado en otros trabajos con tipos de vegetación similares, por ejemplo De Jong et al. (1999) reportó densidades de carbono en biomasa de $145.7 \mathrm{Mg} \mathrm{C}$ ha $^{-1}$; Ordóñez et al. (2008) 169.7 Mg C ha ${ }^{-1}$; Galeana et al. (2013) $163 \mathrm{Mg} \mathrm{C}$ ha $^{-1}$ (bosque cerrado); incluyendo a un bosque mono específico de $A$. religiosa de 75 años de edad ubicado en el Cofre de Perote, Veracruz en el que se estimó una densidad de $\mathrm{C}$ en biomasa aérea arbórea de $157 \mathrm{Mg} \mathrm{C} \mathrm{ha}^{-1}$ (Mendoza y Galicia, 2010). Lo anterior se debe, como ya se mencionó, a que se encontraron

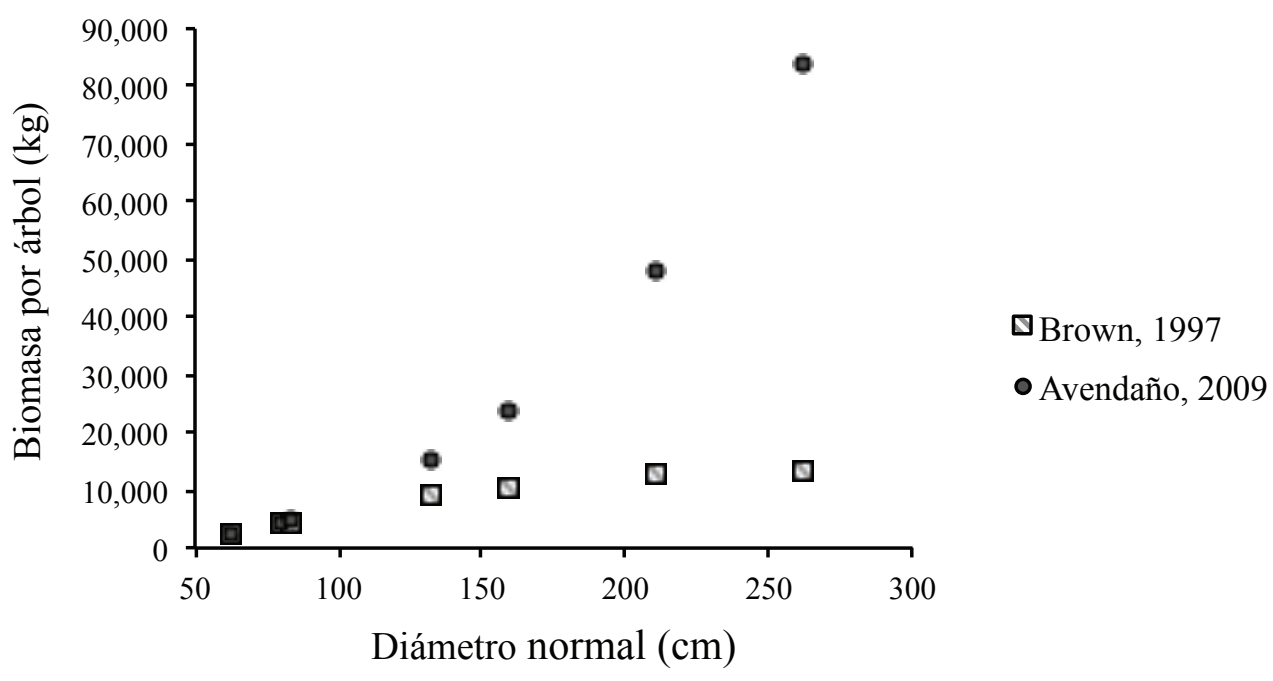

Figura 4. Biomasa aérea arbórea de Abies religiosa, utilizando las ecuaciones alométricas propuestas por Avendaño et al. (2009) y Brown et al. (1997), especímenes con $\mathrm{DN}>60 \mathrm{~cm}$. 
Cuadro 6. Densidad de carbono en todos los depósitos.

\begin{tabular}{|c|c|c|c|c|c|c|c|}
\hline \multirow{2}{*}{ Bosque de referencia } & \multirow{2}{*}{ Sitio } & \multicolumn{6}{|c|}{ Carbono almacenado $\left(\mathrm{Mg} \mathrm{ha}^{-1}\right)$} \\
\hline & & $\begin{array}{c}\text { Biomasa } \\
\text { aérea }\end{array}$ & $\begin{array}{c}\text { Biomasa } \\
\text { subterránea }\end{array}$ & $\mathrm{COS}$ & Mantillo & $\begin{array}{c}\text { Madera } \\
\text { muerta }\end{array}$ & Total \\
\hline \multirow[t]{4}{*}{ Bosque de Abies religiosa } & 1 & 92 & 20 & 80 & 14 & 8 & 214 \\
\hline & 2 & 135 & 27 & 49 & 7 & 7 & 225 \\
\hline & 3 & 280 & 48 & 69 & 10 & 18 & 426 \\
\hline & 4 & 440 & 79 & 74 & 14 & 31 & 637 \\
\hline Media & & 237 & 43 & 68 & 11 & 16 & 376 \\
\hline Desviación estándar & & 158 & 26 & 14 & 4 & 11 & 200 \\
\hline C. $V^{\dagger}(\%)$ & & 67 & 61 & 20 & 31 & 68 & 53 \\
\hline \multirow[t]{4}{*}{ Bosque de Cupressus lusitanica } & 1 & 72 & 18 & 117 & 7 & 3 & 217 \\
\hline & 2 & 73 & 17 & 105 & 5 & 19 & 218 \\
\hline & 3 & 76 & 19 & 99 & 5 & 6 & 205 \\
\hline & 4 & 70 & 18 & 89 & 2 & 1 & 181 \\
\hline Media & & 73 & 18 & 102 & 5 & 7 & 205 \\
\hline Desviación estándar & & 3 & 1 & 12 & 2 & 8 & 17 \\
\hline C. $V^{\dagger}(\%)$ & & 4 & 4 & 11 & 41 & 110 & 9 \\
\hline
\end{tabular}

$\bar{\dagger}=$ coeficiente de variación.

árboles de grandes dimensiones en dos de los sitios de muestreo que integraron el BR1. En contraste, las densidades medias de carbono estimadas en el BR2 para biomasa $\left(73 \mathrm{Mg} \mathrm{ha}^{-1}\right)$ son inferiores a las reportadas por otros autores en tipos de vegetación similares (bosques de pino-encino), por ejemplo, De Jong et al., 1999, reportó densidades de carbono en biomasa de $166.3 \mathrm{Mg} \mathrm{ha}^{-1}$ y Ordóñez et al. (2008) 115.7 $\mathrm{Mg} \mathrm{ha}^{-1}$ ), debido principalmente a que este bosque tuvo especímenes con DN pequeño $(10-40 \mathrm{~cm})$ y a que ha estado sujeto a tala, debido a la gran cantidad de tocones que se midieron, como se reportó en el Cuadro 1.

La densidad del COS de ambos BR se encuentra por debajo de lo reportado por otros autores para vegetación similar en México, tales como De Jong et al. (1999) quienes encontraron densidades de $172.6 \mathrm{Mg} \mathrm{C} \mathrm{ha}{ }^{-1}$ (Bosque de pino) y 174.4 $\mathrm{Mg} \mathrm{C} \mathrm{ha}^{-1}$ (bosque de pinoencino) en los altos de Chiapas; Cairns et al. (1997) reportan una densidad promedio de $118 \mathrm{Mg} \mathrm{C} \mathrm{ha}^{-1}$ para bosques de México. El contenido de $\mathrm{C}$ en este depósito fue mayor en el BR2 que en el BR1, lo que se debe principalmente a que el porcentaje de la fracción gruesa del BR1 es mayor al del BR2, por lo que esta fracción se resta de la masa del suelo, de acuerdo a la Ecuación 4.
En el caso del contenido de $\mathrm{C}$ en Mantillo, los valores encontrados (11 y $\left.5 \mathrm{Mg} \mathrm{ha}^{-1}\right)$ son inferiores al reportado por Woodall (2012), quien encontró que la densidad promedio de $\mathrm{C}$ en este depósito en los ecosistemas forestales de Estados Unidos fue de $25.6 \mathrm{Mg} \mathrm{ha}^{-1}$, lo cual puede deberse a que los sitios medidos en este estudio están sujetos a intervención humana, por lo que es probable que parte del mantillo se haya retirado para utilizarse como combustible (extracción de leña) o materia orgánica para plantas (tierra de monte), prácticas comunes en la zona; sin embargo, esto no fue cuantificado.

\section{CONCLUSIONES}

- Los resultados obtenidos en el presente estudio mostraron que los depósitos de carbono mayores fueron la biomasa viva sobre el suelo (biomasa aérea) y el carbono orgánico del suelo (COS). El primero fue más importante en el bosque de Abies religiosa con $237 \mathrm{Mg} \mathrm{ha}^{-1}$ (representó el 59\% del total de C almacenado) y el segundo fue el mayor depósito en el bosque de Cupressus lusitanica con $102 \mathrm{Mg} \mathrm{ha}^{-1}$ (representó el 50\% del C total estimado). En ambos bosques la suma de estos dos depósitos fue superior al $80 \%$ del total del $\mathrm{C}$ almacenado. Asimismo, dentro del 
depósito correspondiente a la biomasa aérea, el estrato arbóreo resultó ser mucho mayor que el arbustivo y herbáceo (representó más del $98 \%$ en ambos bosques con respecto al total estimado en biomasa aérea).

- De acuerdo a los resultados obtenidos con las ecuaciones alométricas desarrolladas para Abies religiosa, éstas sobreestiman la biomasa aérea y, por lo tanto, su contenido de carbono, con excepción de la desarrollada por Brown et al. (1997). Dado lo anterior, es necesario explorar funciones que representen de manera más adecuada la acumulación de biomasa y carbono en la vegetación arbórea, ya que existen indicios de que las funciones exponenciales representan adecuadamente solo una parte del ciclo de crecimiento, por lo que se concluye que es necesario construir ecuaciones alométricas que representen el ciclo de crecimiento completo, incluyendo árboles de grandes dimensiones.

- Es importante medir los depósitos de carbono que la mayoría de los estudios no estiman: biomasa subterránea, madera muerta sobre el suelo y mantillo; ya que su aporte como depósito de $\mathrm{C}$ aunque no es tan grande como el de biomasa aérea y COS, en conjunto se estimó en este estudio entre 14\% (bosque de Cupressus lusitanica) y $19 \%$ (bosque de Abies religiosa), pero dada la extensión de los bosques en nuestro país, representan un depósito de carbono importante, mismo que debe ser considerado como parte del ciclo global del C. Adicionalmente, la estimación del contenido de $\mathrm{C}$ en estos depósitos representa un avance en el conocimiento del ciclo de este elemento en el sector silvícola; además de que juegan un rol muy importante en la salud del ecosistema, ya que son fuentes primarias de nutrientes y almacén de combustibles para potenciales incendios forestales.

- Finalmente, se encontró que el contenido de $\mathrm{C}$ en el bosque de oyamel fue superior a lo reportado en otros sitios con vegetación similar, por lo que resulta importante conservar este tipo de vegetación como reservorio de carbono en el área de estudio y nos da una idea precisa del potencial como almacén de $\mathrm{C}$ que este tipo de vegetación tiene cuando llega a estados maduros.

\section{LITERATURA CITADA}

Acosta-Mireles, M., J. Vargas-Hernández, A. Velásquez-Martínez y J. D. Etchevers-Barra. 2002. Estimación de la biomasa aérea mediante el uso de relaciones alométricas en seis especies arbóreas en Oaxaca, México. Agrociencia 6: 725-736.
Arriaga, L., J. M. Espinoza, C. Aguilar, E. Martínez, L. Gómez y E. Loa (coordinadores). 2000. Regiones terrestres prioritarias de México. Escala de trabajo 1:1 000 000. Comisión Nacional para el Conocimiento y uso de la Biodiversidad. México.

Avendaño, D., M. Acosta, F. Carrillo y J. Etchevers. 2009. Estimación de la biomasa y carbono en un bosque de Abies religiosa. Fitotec. Mex. 32: 233-238.

Batjes, N. H. 1996. Total carbon and nitrogen in the soils of the world. Eur. J. Soil Sci. 47: 151-163.

Beer, C., M. Reichstein, E. Tomelleri, P. Ciais, M. Jung, N. Carvalhais, C. Rödenbeck, M. A. Arain, D. Baldocchi, G. B. Bonan, A. Bondeau, A. Cescatti, G. Lasslop, A. Lindroth, M. Lomas, S. Luyssaert, H. Margolis, K. W. Oleson, O. Roupsard, E. Veenendaal, N. Viovy, C. Williams, F. I. Woodward, and D. Papale. 2010. Terrestrial gross carbon dioxide uptake: Global distribution and covariation with climate. Science 329: 834-838.

Berg, B. 2000. Litter decomposition and organic matter turnover in Northern forest soils. For. Ecol. Manage. 133: 13-22.

Bolin, B., B. R. Döös, J. Jäger, and R. A. Warrick.1989. The greenhouse effect, climatic change, and ecosystems. Scope No 29. Wiley-Blackwell. Sedona, AZ, USA. ISBN-10: 0471910120.

Brown, S., P. Schroeder, and R. Birdsey. 1997. Aboveground biomass distribution of US eastern hardwood forests and the use of large trees as an indicator of forest development. For. Ecol. Manage. 96: 37-47.

Brown, S. 1997. Estimating biomass and biomass change of tropical forests. FAO Forestry paper 55. Rome, Italy.

Búrquez, A., A. Martínez-Yrízar, S. Núñez, T. Quintero, and A. Aparicio. 2010. Aboveground biomass in three Sonoran Desert communities: Variability within and among sites using replicated plot harvesting. J. Arid Environ. 74: 1240-1247. doi:10.1016/j.jaridenv.2010.04.004.

Cairns, M., S. Brown, E. H. Helmer, and G. A. Baumgardner. 1997. Root biomass allocation in the world's upland forests. Oecologia 111: 1-11.

Caldato, S. L., P. A. Floss y E. C. Serafini Floss. 2010. Producción y descomposición de hojarasca en la selva ombrófila mixta en el sur de Brasil. Bosque 31: 3-8.

Ciais, P., C. Sabine, G. Bala, L. Bopp, V. Brovkin, J. Canadell, A. Chhabra, R. DeFries, J. Galloway, M. Heimann, C. Jones, C. Le Quéré, R.B. Myneni, S. Piao, and P. Thornton. 2013. Carbon and other biogeochemical cycles. Chapter 6. pp. 465-570. In: T. F. Stocker, D. Qin, G. K. Plattner, M. Tignor, S. K. Allen, J. Boschung, A. Nauels, Y. Xia, V. Bex, and P. M. Midgley (eds.). Climate change 2013: The physical science basis. Contribution of working Group I to the fifth assessment report of the intergovernmental panel on climate change Cambridge University Press. Cambridge, UK, New York, NY, USA.

Chojnacky, D. C. and M. Milton. 2008. Measuring carbon in shrubs Chapter 5. pp. 45-72. In: C. M. Hoover (ed.). Field measurements for forest carbon monitoring: A landscape-scale approach. Springer Science + Business Media. New York, NY, USA.

CONAFOR (Comisión Nacional Forestal). 2011. Inventario nacional forestal y de suelos: Manual y procedimientos para el muestreo de campo, re-muestreo 2011. Zapopan, Jal., México.

De Jong, B., M. Cairns, P. Haggerty, N. Ramírez, S. Ochoa, J. Mendoza, M. González, and I. March. 1999. Land-use change 
and carbon flux between 1970s and 1990s in Central Highlands of Chiapas, Mexico. Environ. Manage. 23: 373-385.

De Jong, B., M. Olguín, F. Rojas, V. Maldonado, F. Paz, J. Etchevers, C. Cruz y J. Argumedo. 2009. Actualización del Inventario Nacional de Emisiones de Gases de Efecto Invernadero 19902006 en la Categoría de Agricultura, Silvicultura y otros usos de la tierra. Instituto Nacional de Ecología (INE). México, D. F.

Dixon, R. K., S. Brown, R. A. Houghton, A. M. Solomon, M. C. Trexler, and J. Wisniewski. 1994. Carbon pools and flux of global forest ecosystems. Science 263: 185-190.

Eswaran, H., E. van den Berg, and P. Reich. 1993. Organic carbon soils of the world. Soil Sci. Soc. Am. J. 57: 192-194.

FAO (Food and Agriculture Organization of the United Nations). 2013. GlobAllomeTree-Assessing volume, biomass and carbon stocks of trees and forests. http://www.globallometree. org/data/allometric-equations/ (Consulta: enero 11, 2017).

FAO (Food and Agriculture Organization of the United Nations). 2004. Global forest resources assessment update 2005, Terms and definitions (final version). (http://www.fao.org/ docrep/007/ae156e/ae156e00.HTM). (Consulta: enero 11, 2017).

Flores, P., M. A. López, G. Ángeles, M. de la Isla y G. Calva. 2011. Modelos para estimación y distribución de biomasa de Abies religiosa (Kunth) Schltdl. et Cham. en proceso de declinación. Rev. Mex. Cien. For. 2: 9-20.

Gardi, C., M. Angelini, S. Barceló, J. Comerma, C. Cruz Gaistardo, A. Encina Rojas, A. Jones, P. Krasilnikov, B. Mendonça Santos, L. Montanarella, O. Muñiz, P. Schad, M. Vara Rodríguez y R. Vargas. 2014. Atlas de suelos de América Latina y el Caribe. Comisión Europea - Oficina de Publicaciones de la Unión Europea, L-2995 Luxemburgo. Luxemburgo.

Galeana, J. M., J. A. B. Ordóñez y N. Corona. 2013. Estimación de contenido de carbono en la cuenca del río Magdalena, México. Madera Bosques 19: 53-69.

Harmon, M. E., J. F. Franklin, F. J. Swanson, P. Sollins, S. V. Gregory, J. D. Lattin, N. H. Anderson, S. P. Cline, N. G. Aumen, J. R. Sedell, G. W. Lienkaemper, K. Cromack, and K. W. Cummins. 1986. Ecology of coarse woody debris in temperate ecosystems. Adv. Ecol. Res. 15: 133-302.

Houghton, R. A. y C. M. Woodwell. 1989. Global climate change. Sci. Am. 260: 36-40.

Houghton, R. A., J. I. House, J. Pongratz, G. R. van der Werf, R. S. DeFries, M. C. Hansen, C. Le Quéré, and N. Ramankutty. 2012. Carbon emissions from land use and land-cover change. Biogeosci. Discuss. 9: 835-878. doi:10.5194/bg-9-5125-2012.

Houghton, J. T., L. G. Meira Filho, B. A. Callander, N. Harris, A. Kattenberg, and K. Maskell. 1995. Climate change 1995. The Science of Climate Cliange. Contribution of WGI to the Second Assessment Report of the Intergovernmental Panel on Climate Change. Cambridge University Press. Cambridge, England.

IPCC (Intergovernmental Panel on Climate Change). 2003. Good Practice Guidance for Land Use, Land Use Change and Forestry. IGES. Kanagawa, Japan.

IPCC (Intergovernmental Panel on Climate Change). 2006. IPCC Guidelines for National Greenhouse Gas Inventories. IGES. Kanagawa, Japan.
Keenan, R., C. E. Prescott, and J. P. H. Kimmins. 1993. Mass and nutrient content of woody debris and forest floor in western red cedar and western hemlock forests on northern Vancouver Island. Can. J. For. Res. 23: 1052-1059.

Lal, R. 2003. Soil erosion and the global carbon budget. Environ. Int. 29: 437-450.

Lashof, D. A. and D. R. Ahuja. 1990. Relative contributions of greenhouse gas emissions to global warming. Nature 344: 529-531.

Le Quéré, C., M. R. Raupach, J. C. Canadell, G. Marland, L. Bopp, P. Ciais, T. J. Conway, S. C. Doney, R. A. Feely, P. Foster, P. Friedlingstein, K. Gurney, R. A. Houghton, J. I. House, C. Huntingford, P. E. Levy, M. R. Lomas, J. Majkut, N. Metzl, J. P. Ometto, G. P. Peters, I. C. Prentice, J. T. Randerson, S. W. Running, J. L. Sarmiento, U. Schuster, S. Sitch, T. Takahashi, N. Viovy, G. R. van der Werf, and F. I. Woodward. 2009. Trends in the sources and sinks of carbon dioxide. Nat. Geosci. 2: 831-836.

Masera, O. 1995. Carbon mitigation scenarios for mexican forests: Methodological considerations and results. Interciencia 20: 388-395.

Mendoza, A. and L. Galicia. 2010. Aboveground and belowground biomass and carbon pools in highland temperate forest landscape in Central Mexico. Forestry 83: 497-506. doi:10.1093/forestry/cpq032.

Moctezuma Barragán, P. e I. Carreón. 2005. Sembrando el futuro en la Sierra Nevada. http://colectivopalabra.com/sembrandoel-futuro-en-la-sierra-nevada (Consulta: agosto 30, 2016).

OECD (Organisation for Economic Co-operation and Development). 2015. Territorial reviews: Valle de México, Mexico. OECD Publishing. Paris. http://dx.doi. org/10.1787/9789264245174-en.

Ordóñez, J. A. B., B. H. J. De Jong, F. García-Oliva, F. L. Aviña, J. V. Pérez, G. Guerrero, R. Martínez, and O. Masera. 2008. Carbon content in vegetation, litter, and soil under 10 different land-use and land-cover classes in the Central Highland of Michoacán, Mexico. For. Ecol. Manage. 255: 2074-2084.

Prentice, I. C., G. D. Farquhar, M. J. R. Fasham, M. L. Goulden, M. Heimann, V. J. Jaramillo, H. S. Kheshgi, C. Le Quéré, R. J. Scholes, and D. W. R. Wallace. 2001: The carbon cycle and atmospheric carbon dioxide. pp. 99-181. In: J. T. Houghton, Y. Ding, D. J. Griggs, M. Noguer, P. J. van der Linden, X. Dai, K. Maskell, and C. A. Johnson (eds.). Climate change 2001: The scientific basis. Contribution of Working Group I to the Third Assessment Report of the Intergovernmental Panel on Climate Change. Cambridge University Press. Cambridge, UK.

Rodríguez, V. y G. Calva. 2013. Estimación del carbono contenido en el bosque de Abies religiosa (H.B.K.) Schl. et Cham. en el Parque Nacional "El Chico", Hidalgo. pp. 7-13. In: F. Paz Pellat, J. Wong G., M. Bazan y V. Saynes (eds.). Estado actual del conocimiento del ciclo del carbono y sus interacciones en México, Síntesis a 2013. PMC. Texcoco, México. ISBN 978607-96490-1-2.

Rojas G., F. y L. Villers R. 2008. Estimación de la biomasa forestal del Parque Nacional Malinche: Tlaxcala-Puebla. Rev. Cienc. For. Méx. 33: 59-86.

Sánchez G., A. y L. López M. 2003. Clasificación y ordenación de la vegetación del norte de la Sierra Nevada, a lo largo de un gradiente altitudinal. UNAM. Anal. Inst. Biol. Serie Botánica 74: 47-71. 
Schaetzl, R. J., S. F. Burns, D. Johnson, and T. Small. 1989. Tree uprooting: Review of impacts on forest ecology. Vegetation 79: $165-176$

Schimel, D. S. 1995. Terrestrial ecosystems and the carbon cycle. Global Change Biol. 1: 77-91.

Schneider, S. H. 1989. The greenhouse effect: Science and policy. Science 243: 771-781.

Schulze, E. D., C. Wirth, and M. Heimann. 2000. Managing forest after Kyoto. Science 289: 2058-2059.

SEMARNAT-INECC (Secretaría de Medio Ambiente y Recursos Naturales e Instituto Nacional de Ecología y Cambio Climático). 2009. México Cuarta Comunicación Nacional ante la Convención Marco de las Naciones Unidas sobre el Cambio Climático. México, D. F. ISBN: 978-607-7908-00-5.
SEMARNAT-INECC (Secretaría de Medio Ambiente y Recursos Naturales e Instituto Nacional de Ecología y Cambio Climático). 2012. México Quinta Comunicación Nacional ante la Convención Marco de las Naciones Unidas sobre el Cambio Climático. México, D. F. ISBN: 978-607-824-650-2.

Swift, M. J., O. W. Heal, and J. M. Anderson. 1979. Decomposition in terrestrial ecosystems. Estudios de Ecología, Vol. 5. University of California Press. Los Ángeles, CA, USA.

Van Wagner, C. E. 1968. The line intersect method in forest fuel sampling. For. Sci. 14: 20-26.

Woodall, C. W., C. H. Perry, and J. A. Westfall. 2012. An empirical assessment of forest floor carbon stock components across the United States. For. Ecol. Manage. 269: 1-9. 